\title{
DESEMPENHO DE ESPÉCIES DE Eucalyptus spp. NA REGIÃO SEMI-ÁRIDA DO PLANALTO DE CONQUISTA NA BAHIA, BRASIL
}

\author{
'Adalberto Brito de Novaes, ${ }^{2}$ Thiago Alves Santos de Oliveira, ${ }^{2}$ Saulo Alves Santos de \\ Oliveira, ${ }^{3}$ Alexandre Alves Bomfim.
}

\section{SUMMARY}

The present study has anlysed the performance of Eucalyptus citriodora; E. tereticornis; E. urophilla; E. camaldulensis; E. robusta; E. cloeziana; and E. urograndis in region of Bosque Estacional Decidual do Sales in the State Bahia into the southwest region of the State of Bahia, Brazil. The species mentioned above had been adopted 7 corresponding treatments. The evaluation of the performance of the plants in the field, to the 15 months of the planting, consisted of the measurement of the height of the aerial part and diameter to the level of the ground. For all the gotten results, the averages of the treatments had been compared by the test of Duncan with the level of $95 \%$ of probability. In accordance with the gotten results are concluded that Eucalyptus tereticornis and Eucalyptus camaldulensis species had presented the biggest values of height and diameter, being considered apt to integrate projects of reforestation in the region.

Key words: Eucalyptus tereticornis Eucalyptus camaldulensis, height, diameter.

'Prof . DS.Titular do D.F.Z., Universidade Estadual do Sudoeste da Bahia. Brasil. adalbert@uesb.br.

${ }^{2}$ Bolsista FAPESB. Vitória da Conquista-BA. Brasil. saulowarrior@yahoo.com.br

${ }^{3}$ Mestrando em Agronomia-UESB. Vitória da Conquista-BA. floresteiro2000@hotmail.com) 


\section{RESUMO}

O presente estudo teve como objetivo, avaliar o desempenho de Eucalyptus citriodora; E. tereticornis; E. urophilla; E. camaldulensis; E. robusta; E. urograndis e E. cloeziana, em região de Floresta Estacional Decidual no município de Cândido Sales na região Sudoeste da Bahia, Brasil. Foram adotados 7 tratamentos correspondentes as espécies acima mencionadas. A avaliação do desempenho das plantas no campo, aos 15 meses do plantio, constou da mensuração da altura da parte aérea e diâmetro ao nivel do solo, efetuadas por meio de vara graduada e paquímetro digital, respectivamente. Para todos os resultados obtidos, as médias dos tratamentos foram comparadas pelo teste de Duncan ao nível de $95 \%$ de probabilidade. De acordo com os resultados obtidos conclui-se que as espécies, Eucalyptus tereticornis e $E$. camaldulensis apresentaram os maiores valores de altura e diâmetro, sendo consideradas aptas para integrarem projetos de reflorestamento na região.

Palavras-chave: Eucalyptus tereticornis Eucalyptus camaldulensis, altura, diâmetro.

\section{RESUMEN}

Em el presente trabajo es analizado el comportamiento de Eucalyptus citriodora, $E$. tereticornis, E. urophilla, E. camaldulensi, E. robusta, E. urograndis y E. cloeziana en la región del Bosque Estacional Decidual en el Municipio de Cândido Sales en el suroeste de Bahia, Brasil. Se midió altura total y diámetro al nivel del suelo a los 115 meses de edad de las plantaciones. Las medias obtenidas fueron comparadas mediante el tes de Duncan a un nivel de probabilidad del $95 \%$. Se concluye que las especies Eucalyptus tereticornis y $E$. camaldulensis presentan los mayores valores de altura y diámetro y son consideradas aptas para la forestación en la región.

Palabras claves: Eucalyptus tereticornis Eucalyptus camaldulensis, altura, diámetro. 


\section{INTRODUÇÃO}

Apesar dos poucos remanescentes florestais existentes na Bahia, é muito grande a sua dependência em produtos e subprodutos oriundos das florestas plantadas. O Planalto de Conquista, localizado na Região do Sudoeste da Bahia, apresenta uma vegetação típica denominada de "Mata de Cipó" que outrora, cobria toda a extensão do Sudeste da Bahia. Muitas dessas áreas foram desmatadas, sem que nenhuma outra atividade ocupasse o solo, fazendo com que o mesmo ficasse desprotegido sem produzir alimentos, fibras e/ou energia para a regiāo. Este processo vem intensificando o êxodo rural, a fome de quem permanece na terra improdutiva por limitações diversas (Novaes et al., 1992).

Nesta ótica, o reflorestamento auto-sustentável, com espécies florestais de rápido crescimento, a exemplo do Eucalipto, justifica-se na medida em que, trata-se de uma das poucas opções de fato viável, que possibilitará não só a preservação de nossas matas remanescentes, como também a geraçāo de riquezas, empregos diretos e indiretos, além de fomentar a implantação de indœstrias na região, gerando renda e novos postos de empregos (Novaes et al.,1992).

Para Drumond (1992), no Nordeste brasileiro destaca-se a região entre os municipios de Ouricuri-PE e Arariopina onde existe uma concentração de indœstrias que utilizam a lenha como fonte de energia, consumindo em média $30 \mathrm{mil} \mathrm{m}^{3}$ de lenha por mês. $\mathrm{O}$ uso do eucalipto em programas de reflorestamento é uma consequência dessa árvore apresentar rápido crescimento, alem de uma diversidade de espécies diferentes, cerca de 600 espécies, distribuidas no continente australiano de onde se originou e difundidas em mais de 100 paises no mundo. São espécies que primam pela versatilidade, vegetando nas mais diferentes condições de solo e clima. De acordo com Golfari (1978), a escolha correta de espécies e procedências adequadas para uma região, pode ser feita somente por meio da experimentação e ainda, a existência efetiva numa região, de parcelas experimentais ou plantios em idade adulta, representa a base mais segura para determinar o grau de adaptação de uma espécie, estimar sua produtividade e avaliar o potencial ecológico da área.

Coutinho et al. (2004), trabalhando com espécies de eucalipto na região da Zona da Mata de Pernambuco, reportaram que o E. citriodora, E. urophylla, E. camaldulensis, E. saligna, E. grandis e E. dunnii apresentaram as melhores performances de altura e diâmetro. Este trabalho objetivou avaliar o comportamento das espécies Eucalyptus citriodora, E. tereticomis, E. urophilla, $E$. camaldulensis, E. robusta, E. urograndis e E. cloeziana na região semi-árida do Planalto de Conquista na região Sudoeste da Bahia, no município de Candido Sales - BA, visando selecionar espécies com potencial para integrar projetos de reflorestamento na região.

\section{MATERIAL E MÉTODOS}

O presente estudo foi desenvolvido na Fazenda Ouro Verde, no município de Candido Sales-BAA região apresenta indice pluviométrico variando de 500 a $850 \mathrm{~mm}$ anuais, sendo os meses mais chuvosos de novembro a março. A temperatura média anual é de $22^{\circ} \mathrm{C}$. O solo da área foi classificado como LVA distrófico, com textura média e topografia suavemente ondulada a plana e boa drenagem. A vegetação predominante corresponde a Floresta Estacional Decidual. 
Foram adotados 7 tratamentos correspondentes as espécies, Eucalyptus citriodora, E. tereticornis, E. urophilla, E. camaldulensis, E. robusta, E. urograndis e E. cloeziana. O delineamento experimental utilizado foi em blocos ao acaso, com 4 repetições e parcelas constituidas de 30 plantas, perfazendo um total de 2.520 no experimento. As mudas foram produzidas em tubetes com dimensões de $12,5 \mathrm{~cm}$ altura, $3 \mathrm{~cm}$ com capacidade volumétrica de $50 \mathrm{~cm}^{3}$. O substrato utilizado constituiu de $50 \%$ de vermiculita, $40 \%$ de composto orgânico e $10 \%$ de moinha de carvão. No campo após o preparo do solo, as mudas foram plantadas no espaçamento 3,0 × 3,0 m e adubadas com $200 \mathrm{~g}$ de adubo Superfosfato Simples.

A avaliação do desempenho das plantas no campo, aos 15 meses do plantio, constou da mensuração da altura da parte aérea e diâmetro ao nivel do solo, efetuadas por meio de vara graduada e paquímetro digital, respectivamente. Para todos os resultados obtidos, as médias dos tratamentos foram comparadas pelo teste de Duncan ao nivel de $95 \%$ de probabilidade.

\section{RESULTADOS E DISCUSSÃO}

Na Tabela $N^{\circ} 1$ estão os dados de sobrevivência das espécies utilizadas no presente estudo. Os maiores índices de sobrevivência foram verificados para as espécies Eucalyptus camaldulensis e Eucalyptus tereticornis. O menor índice coube a espécie Eucalyptus citriodora.

\section{Tabela $N^{\circ} 1$ \\ TAXA DE SOBREVIVÊNCIA DAS ESPÉCIES TRÊS MESES APÓS O PLANTIO}

\begin{tabular}{|l|c|}
\hline \multicolumn{1}{|c|}{ Espécie } & Sobrevivência (\%) \\
\hline Eucalyptus camaldulensis & 94,20 \\
\hline Eucalyptus tereticornis & 93,26 \\
\hline Eucalyptus urograndis & 87,12 \\
\hline Eucalyptus robusta & 85,49 \\
\hline Eucalyptus urophylla & 83,29 \\
\hline Eucalyptus cloeziana & 82,89 \\
\hline Eucalyptus citriodora & 80,33 \\
\hline
\end{tabular}

Quanto ao desempenho das mudas no campo, 15 meses após o plantio, verificou-se que as espécies Eucalyptus camaldulensis e Eucalyptus tereticornis, apresentaram as maiores médias de altura da parte aérea, sendo constatada pelo teste de Duncan (95\%), diferença significativa em relação as demais (Tabela $N^{\circ} 2$ ). Este resultado, obtido neste período, aponta essas espécies como potenciais para serem utilizadas em programas de reflorestamento na região, embora, esses resultados possam apresentar alterações ao final do período de crescimento inicial (dois anos). Quanto ao diâmetro ao nível do solo, neste mesmo período, constatou-se que o Eucalyptus tereticornis, apresentou a maior média, diferindo significativamente pelo teste de Duncan (95\%), dos demais tratamentos, mostrando assim, que esta espécie, provavelmente, poderá apresentar maior volumetria no final da rotação. Autores como McGilvray e Barnett (1981) e Barnett (1990) destacaram a altura como um parâmetro de maior correlação com o desempenho das mudas no campo, todavia, Barnett (1990) destacou, ainda, a necessidade de um diâmetro de colo compatível com a altura para que o desempenho no campo corresponda às expectativas. 
Tabela $\mathbf{N}^{\circ} 2$

VALORES MÉdIOS DE ALTURA DA PARTE AÉREA E DIÂMETRO AO NIVEL DO SOLO DE MUDAS 15 MESES APÓS O PLANTIO

\begin{tabular}{|l|l|r|}
\hline \multicolumn{1}{|c|}{ Espécie } & Altura $(\mathrm{m})$ & Diâmetro $(\mathrm{cm})$ \\
\hline Eucalyptus tereticornis & $2,84 \mathrm{a}$ & $5,33 \mathrm{a}$ \\
\hline Eucalyptus camaldulensis & $2,81 \mathrm{ab}$ & $5,09 \mathrm{ab}$ \\
\hline Eucalyptus urograndis & $2,57 \mathrm{abcd}$ & $4,19 \mathrm{abc}$ \\
\hline Eucalyptus citriodora & $2,54 \mathrm{abcd}$ & $4,13 \mathrm{abc}$ \\
\hline Eucalyptus urophylla & $2,49 \mathrm{abcd}$ & $3,47 \mathrm{abc}$ \\
\hline Eucalyptus robusta & $2,44 \mathrm{~cd}$ & $2,69 \mathrm{abc}$ \\
\hline Eucalyptus cloeziana & $2,36 \mathrm{~d}$ & $2,57 \mathrm{abc}$ \\
\hline
\end{tabular}

Médias seguidas de pelo menos uma mesma letra, não diferem entre si, ao nivel de $5 \%$ de significância, pelo teste de Duncan.

\section{CONCLUSÕES}

De acordo com os resultados obtidos e nas condições em que foi desenvolvido o presente estudo, levando-se em consideração o período no qual foi avaliado o experimento, conclui-se que as espécies Eucalyptus tereticornis e E. camaldulensis foram consideradas aptas para serem utilizadas em projetos de reflorestamento na região.

\section{REFERÊNCIAS}

Barnett, J. P., 1990. Effects of morphological grade on field performance of container-grown southern pines seedlings. Separata de: Biennial Silvicultural Research Conference (6.: 1990, Menphis). Proceedings. Asheville: Southern Forest Experiment Station, 1:94-99. (Gen. Te ch. Rep. SO. USDA For. Serv., n.70).

Coutinho, J. L. B. et al., 2004. Performance of Eucalyptus spp. seedlings in the "dry rainforest" region of the State of Pernambuco, Brazil. I: Results of the first year. Viçosa. Rev. çrvore. vol.28, no.6, p.771-775.

Golfari, L. e Caser, R. L., 1977. Zoneamento ecológico da região Nordeste para experimentação florestal. Belo Horizonte: Centro de Pesquisa Florestal do Cerrado, 116p.

Drumond, M. A., 1992. Reflorestamento na região semi-árida do nordeste brasileiro. In:. NOVAES et al. Reflorestamento no Brasil.. Vitória da Conquista-BA, UESB.p.28-34

McGilvray, J.M., Barnett, J. P., 1981. Relating seedling morphology to field performance of containerized southern pines. Separata de: Southern Containerized Forest Tree Seed Conferece, (Savannah). Proceedings. New Orleans: USDA, For. Serv. Forest Experiment Station, p.39-46.

Novaes, A. B., 1992. Reflorestamento no Brasil. Vitória da Conquista - BA: UESB, 1992, p.175. 
